\title{
Photometric study of two novae in M 31
}

\author{
Y. C. Joshi ${ }^{1}$, A. K. Pandey ${ }^{1}$, D. Narasimha ${ }^{2}$, Y. Giraud-Héraud ${ }^{3}$, R. Sagar ${ }^{1}$, and J. Kaplan ${ }^{3}$ \\ 1 State Observatory, Manora peak, Nainital 263129, Uttaranchal, India \\ 2 Tata Institute of Fundamental Research, Homi Bhabha Road, Mumbai 400 005, India \\ ${ }^{3}$ Laboratoire de Physique Corpusculaire, Collège de France, Laboratoire associé au CNRS-IN2P3 (URA 6411), \\ 11 place Marcelin Berthelot, 75231 Paris Cedex 05, France
}

Received 26 May 2003 /accepted 15 October 2003

\begin{abstract}
We report $R_{\mathrm{c}} I_{\mathrm{c}}$ light curves of 2 novae in the M 31 galaxy which were detected in the four year Nainital Microlensing Survey. One of these novae has been tracked from the initial increase in flux while other has been observed during its descending phase of brightness. The photometry of the first nova during the outburst phase suggests its peak $R_{\mathrm{c}}$ magnitude to be about 17.2 mag with a flux decline rate of $0.11 \mathrm{mag} \mathrm{day}^{-1}$ which indicates that it was a fast nova. A month after its outburst, it shows reddening followed by a plateau in $I_{\mathrm{c}}$ flux. The second nova exhibits a bump in $R_{\mathrm{c}}$ and $I_{\mathrm{c}}$, possibly about three weeks after the outburst.
\end{abstract}

Key words. galaxies: individual: M31 - stars: novae, cataclysmic variables

\section{Introduction}

Cataclysmic variables (CVs) are close binary systems consisting of a white dwarf primary and a late-type main sequence secondary star. Novae are a sub-class of cataclysmic variables characterized by the presence of a sudden increase of brightness, called outbursts, due to thermonuclear runway in the envelope of the primary, causing the system brightness to increase typically by 10-20 mag. These are bright objects which reach up to $M_{V} \sim-9.0 \mathrm{mag}$ at maximum and their rate of decline is tightly correlated with their absolute magnitude at maximum (McLaughlin 1945). The study of novae in external galaxies is important to infer their distances as these objects are one of the brightest standard candles up to the Virgo cluster (cf. Jacoby et al. 1992 for a review) and tracers of differences in the stellar content among galaxies (cf. van den Bergh 1988 for a review).

M31, our nearest large galaxy, has been a target of searches for novae since the pioneering work of Hubble (1929). Later Arp (1956), Rosino (1964, 1973), Rosino et al. (1989), Ciardullo et al. (1987), Sharov \& Alksnis (1991), Tomaney \& Shafter (1992), Rector et al. (1999) and Shafter \& Irby (2001) have extended the systematic search for novae in M 31 . In collaboration with the AGAPE (Andromeda Gravitational Amplification Pixel Experiment) group, we started Cousins $R$ and $I$ photometric observations of M 31 in 1998 to search for microlensing events. Based on the 4 year observations, we have already reported the discovery of new Cepheids and other variable stars (Joshi et al. 2003a). As a microlensing survey program is ideally suited to monitor the flux and temperature

Send offprint requests to: Y. C. Joshi, e-mail: yogesh@upso.ernet.in variation during transient events, we have extended our search to detect nova outbursts. Here we report photometric light curves of two novae detected in the target field, one each in 2000 and 2001 observing seasons. We show that an increase in flux at longer wavelengths a few weeks after the initial rapid decline is a common phenomenon in the novae light curves.

\section{Observations}

We have undertaken a program called the Nainital Microlensing Survey to detect microlensing events in the direction of M31, at the State Observatory, Nainital, India, since 1998. Cousins $R$ and $I$ broad band CCD observations of M31 were carried out for an $\sim 6^{\prime} \times 6^{\prime}$ field in 1998 and an $\sim 13^{\prime} \times 13^{\prime}$ field during 1999 to 2001 observing seasons using the 104-cm Sampurnanand Telescope at the $\mathrm{f} / 13$ Cassegrain focus. The total integrated observing time devoted to the survey ranges from $\sim 30$ min to 2 hours each night. The $13^{\prime} \times 13^{\prime}$ target field $\left(\alpha_{2000}=0^{\mathrm{h}} 43^{\mathrm{m}} 38^{\mathrm{s}}\right.$ and $\delta_{2000}=+41^{\circ} 09$ ' 1 ) is centered at a distance of $\sim 15$ arcmin away from the center of M31. The average seeing during the 141 observed nights spanning 4 years was $\sim 2$ arcsec. An overview of the observational detail has been given by Joshi et al. (2003a).

A large database collected during the observing period was planned as:

(a) to search for microlensing events;

(b) to search for variable stars, particularly Cepheids;

(c) to search for other transient events e.g. novae, where a decrease in brightness of 2 to 5 mag within the first two months of the nova evolution can easily be detected in our observations. 

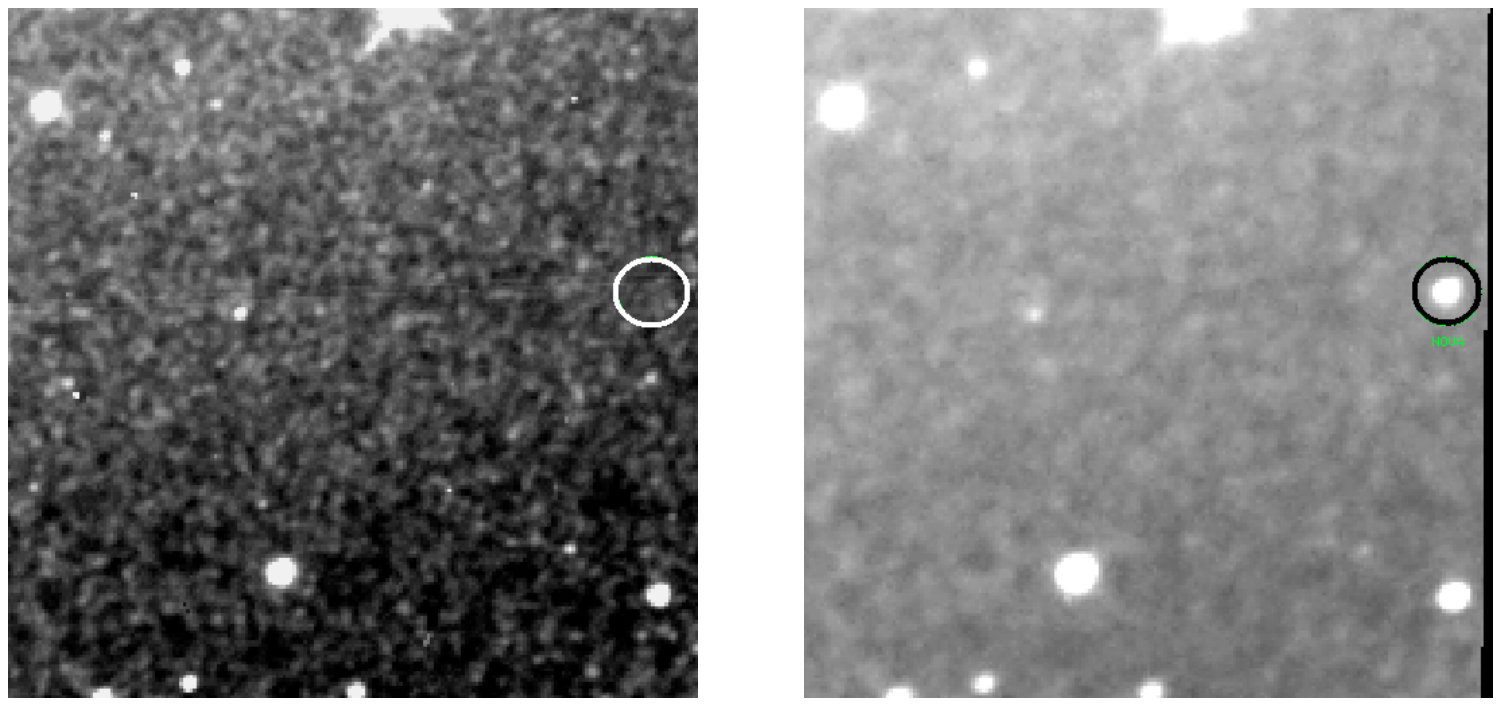

Fig. 1. A 2 arcmin wide subset of two different $R$ band images taken on January 11, 2000 (left panel) and October 20, 2000 (right panel). North is at the top and East is to the left. The left frame shows no star at the position marked by a circle while right frame shows a star (nova NMS-1) of brightness $R \sim 17.2 \mathrm{mag}$ at that position.

We have already published a catalog of variable stars which contains data for 26 Cepheids and 333 red variable stars (Joshi et al. 2003a). We are in the process of analysing some of the possible microlensing candidates found in our data set (Joshi et al. 2003b,c). Here we report the photometry of two novae detected in the target field.

\section{Data reduction and photometry}

Standard techniques were used for data reduction using MIDAS and IRAF software. The dark current correction was not applied due to its negligible contribution during the maximum exposure time of a frame. Cosmic rays (CRs) were removed from each frame independently. We added all the frames of a particular filter taken on a single night and made one frame per filter per night to increase the signal to noise ratio. The CCD frames were processed using the DAOPHOT photometry routine (Stetson 1987). To obtain $R$ and $I$ standard magnitudes, the photometric calibration was done using Landolt's (1992) standard field SA98, on a good photometric night of 25/26 October, 2000. A total of 13 secondary stars having $0.09 \leq(R-I) \leq 1.0$ were observed over a wide range of airmasses. The typical error in magnitudes at $\sim 20.0$ mag level is $\sim 0.10$ and $0.15 \mathrm{mag}$ in $R$ and $I$ bands respectively. In order to use observations in non-photometric conditions, differential photometry was done assuming that the errors introduced due to colour difference between nova and comparison stars were much smaller than the zero point errors. Further details of the photometric calibration has been given in Joshi et al. (2003a).

\section{Identification of the novae}

We identified two novae in the target field while searching for microlensing events using the pixel technique which was initially proposed to detect microlensing events by Baillon et al. (1993). The implementation of the pixel technique in our data is described in detail by Joshi et al. (2001, 2003c). The two novae detected in our survey, one in 2000 and the other in 2001 observing seasons, are named nova NMS-1 and nova NMS-2 where NMS is an acronym for our project "Nainital Microlensing Survey". The two novae are individually discussed in the following subsections.

\subsection{Nova NMS-1}

The nova NMS-1 having celestial coordinates $\alpha_{2000}=$ $00^{\mathrm{h}} 42^{\mathrm{m}} 57^{\mathrm{s}} .1$ and $\delta_{2000}=+41^{\circ} 07^{\prime} 15^{\prime \prime} \cdot 7$ was reported in the IAU circular by Donato et al. (2001). When we started observations at 18:54 UT on October 18, 2000, the nova was still brightening. We followed it through the 2000-2001 observing season. Figure 1 shows two images where the nova is unresolved and at maximum brightness.

The photometry of nova NMS-1 has been carried out using the PSF profile fitting technique. Since it was sufficiently bright during the early phase of its eruption and gradually increased its brightness, we carried out photometry on each individual frame of the observations of 18 and 20 October, 2000 (see Table 1). The light curve of nova NMS-1 is shown in Fig. 2. The brightness of the nova increased by $\sim 0.10 \mathrm{mag}$ in the $R$ band from 18:54 UT to $19: 17$ UT and by $\sim 0.23 \mathrm{mag}$ in $I$ band from 19:35 UT to 20:07 UT. From a comparison of individual frames taken on that day, it appears that we have captured nova NMS-1 during its peak brightness. The total brightness of the nova increased by $\sim 1.5$ mag during October 18-20, 2000 followed by an almost exponential decay. Its rate of decline, $v_{\mathrm{d}}$, defined as the rate in mag day ${ }^{-1}$ at which a nova drops to two magnitudes below maximum brightness, is estimated to be:

$v_{\mathrm{d}, R} \sim 0.11 \mathrm{mag} \mathrm{day}^{-1}$

$v_{\mathrm{d}, I} \sim 0.11 \mathrm{mag}^{-1} \mathrm{day}^{-1}$

for $R$ and $I$ bands respectively. The observed values of $v_{\mathrm{d}}$ suggest that the nova NMS-1 was a fast nova. The maximum 
Table 1. The $R, I$ magnitudes of the nova NMS-1 during outburst. The fractional value for the Julian date is given for those points where we calibrated the magnitude for each individual frame of that night.

\begin{tabular}{cccc|ccc}
\hline \hline $\begin{array}{c}\text { JD } \\
(+2450000)\end{array}$ & $\begin{array}{c}R \\
(\mathrm{mag})\end{array}$ & $\begin{array}{c}\text { JD } \\
(+2450000)\end{array}$ & $\begin{array}{c}I \\
(\mathrm{mag})\end{array}$ & $\begin{array}{c}\text { JD } \\
(+2450000)\end{array}$ & $\begin{array}{c}R \\
(\mathrm{mag})\end{array}$ & $\begin{array}{c}I \\
(\mathrm{mag})\end{array}$ \\
\hline 1836.287 & $18.69 \pm 0.02$ & 1836.316 & $18.38 \pm 0.03$ & 1869 & $19.64 \pm 0.03$ & $19.32 \pm 0.06$ \\
1836.292 & $18.64 \pm 0.02$ & 1836.321 & $18.38 \pm 0.02$ & 1870 & $19.67 \pm 0.02$ & $19.23 \pm 0.05$ \\
1836.296 & $18.60 \pm 0.02$ & 1836.327 & $18.36 \pm 0.02$ & 1872 & $19.69 \pm 0.03$ & $19.39 \pm 0.05$ \\
1836.299 & $18.57 \pm 0.02$ & 1836.333 & $18.36 \pm 0.02$ & 1874 & $19.80 \pm 0.02$ & $19.42 \pm 0.05$ \\
1836.303 & $18.59 \pm 0.02$ & 1836.338 & $18.15 \pm 0.05$ & 1877 & $19.84 \pm 0.03$ & $19.34 \pm 0.07$ \\
1838.213 & $17.19 \pm 0.01$ & 1838.236 & $16.97 \pm 0.01$ & 1878 & $19.93 \pm 0.02$ & $19.24 \pm 0.06$ \\
1838.218 & $17.16 \pm 0.01$ & 1838.245 & $16.95 \pm 0.01$ & 1879 & $20.00 \pm 0.03$ & $19.44 \pm 0.08$ \\
1838.224 & $17.19 \pm 0.01$ & 1838.250 & $16.94 \pm 0.01$ & 1881 & $19.97 \pm 0.02$ & $19.70 \pm 0.06$ \\
- & - & 1838.255 & $16.96 \pm 0.01$ & 1884 & $20.13 \pm 0.02$ & $19.55 \pm 0.05$ \\
- & - & 1838.259 & $16.94 \pm 0.01$ & 1888 & $19.97 \pm 0.07$ & $19.35 \pm 0.08$ \\
1843 & $17.74 \pm 0.01$ & & $17.27 \pm 0.01$ & 1890 & $20.18 \pm 0.06$ & $19.37 \pm 0.05$ \\
1845 & $18.09 \pm 0.02$ & & $17.67 \pm 0.02$ & 1905 & $20.88 \pm 0.06$ & $19.72 \pm 0.05$ \\
1851 & $18.69 \pm 0.02$ & & $18.44 \pm 0.03$ & 1915 & $20.60 \pm 0.12$ & $19.40 \pm 0.12$ \\
1853 & $18.84 \pm 0.02$ & & $18.54 \pm 0.05$ & 1918 & & - \\
1855 & $19.04 \pm 0.02$ & & $18.71 \pm 0.04$ & 1921 & $20.82 \pm 0.08$ & $19.40 \pm 0.06$ \\
1862 & $19.20 \pm 0.03$ & & $18.89 \pm 0.04$ & 1924 & $20.96 \pm 0.07$ & $19.56 \pm 0.04$ \\
1863 & $19.35 \pm 0.03$ & & $19.03 \pm 0.04$ & 1925 & $20.81 \pm 0.06$ & $19.52 \pm 0.04$ \\
1866 & $19.57 \pm 0.02$ & & $19.29 \pm 0.07$ & 1926 & $20.85 \pm 0.08$ & - \\
1867 & $19.61 \pm 0.03$ & & $19.09 \pm 0.04$ & 1927 & $21.28 \pm 0.09$ & $19.50 \pm 0.07$ \\
1868 & $19.68 \pm 0.02$ & & $19.19 \pm 0.06$ & 1929 & $21.63 \pm 0.11$ & - \\
\hline
\end{tabular}

magnitude of the nova NMS-1 at peak brightness is $M_{R}(\max ) \sim$ -7.96 and $M_{I}(\max ) \sim-8.02 \mathrm{mag}$ in $R$ and $I$ bands respectively. Here we consider a true distance modulus of $24.49 \mathrm{mag}$ for M31 and a total extinction of $0.63 \mathrm{mag}$ and $0.47 \mathrm{mag}$ towards our observed direction in the $R$ and $I$ bands respectively (cf. Joshi et al. 2003a). The correlation between observed $R_{\max }$ and rate of decline is consistent with that illustrated by Capaccioli et al. (1989) for novae in M 31.

\subsection{Nova NMS-2}

The nova NMS-2 was already reported in the IAU circular by Li (2001). Figure 3 shows two images where the nova is at unresolved and maximum brightness phase. The celestial coordinates of the nova NMS-2 are $\alpha_{2000}=00^{\mathrm{h}} 43^{\mathrm{m}} 03^{\mathrm{s}} .3$ and $\delta_{2000}=+41^{\circ} 12^{\prime} 10^{\prime}{ }^{\prime} 8$. Since this nova was situated at the edge of our target field, it could not be observed in all the images. When we started our observation on October 12, 2001, it was already in a descending phase of brightness.

Photometry of the nova was carried out during the brighter phase of outburst. We give its $R$ and $I$ magnitudes in Table 2 and the light curve is shown in Fig. 4. A variation of more than 1.5 mag in $R$ band is seen during the first 35 days of its observations. For the nova NMS-2, our photometry is insufficient to characterize its speed class since we do not have any information of its maximum brightness. The brightest magnitude of the nova NMS-2 in our observations is estimated to

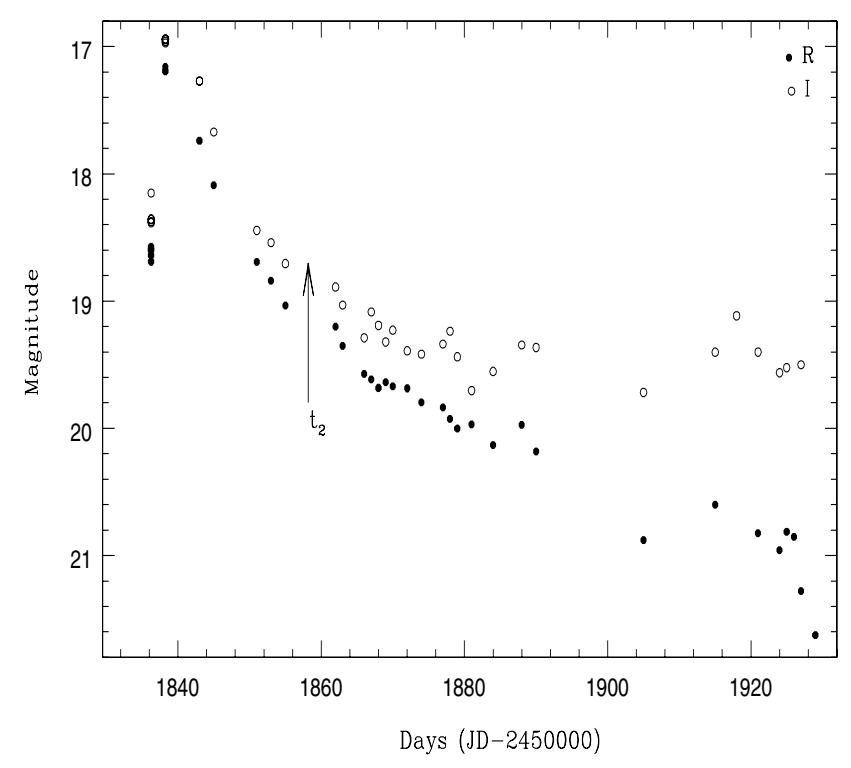

Fig. 2. $R, I$ light curves of the nova NMS-1 during outburst where filled and open circles represent $R$ and $I$ magnitudes respectively. The arrow indicates the time where the nova falls 2 mag below its maximum brightness.

be $\sim 17.7 \mathrm{mag}$ in both $R$ and $I$ bands indicating that it must be a star brighter than $M_{R}, M_{I} \sim-7.4$ mag during its peak brightness. 

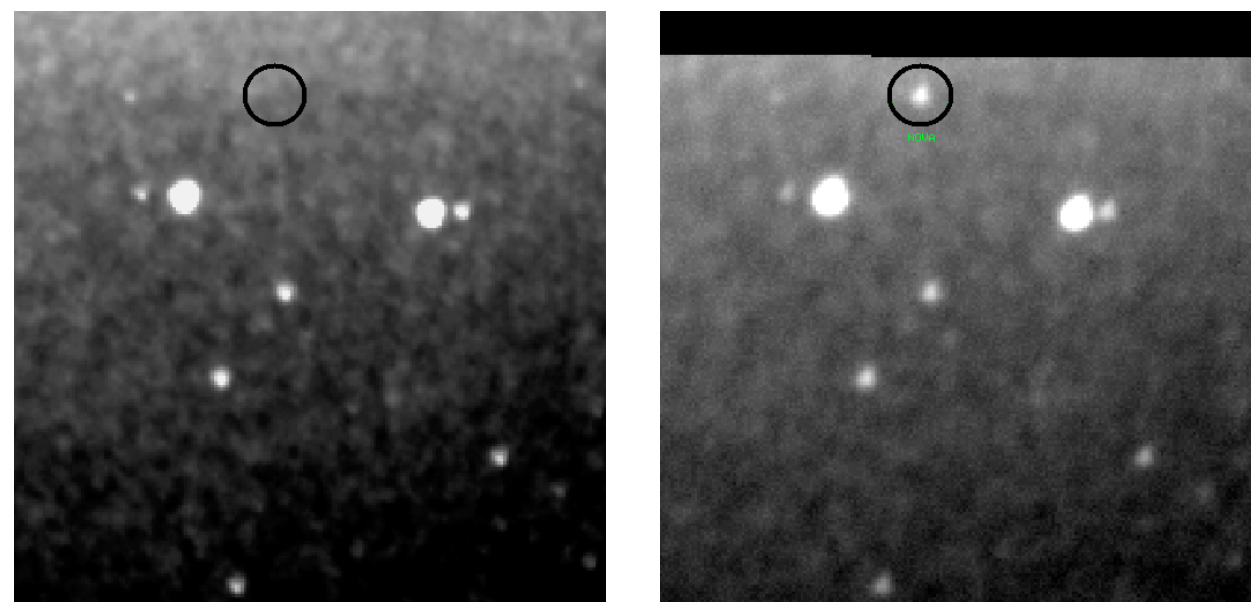

Fig. 3. A 2 arcmin wide subset of two different $R$ band images taken on January 17, 2001 (left panel) and October 19, 2001 (right panel). North is at the top and East is to the left. The left frame shows no star at the position marked by a circle while right frame shows a star (nova NMS-2) at that position of brightness $R \sim 17.7 \mathrm{mag}$.

Table 2. The $R, I$ magnitudes of the nova NMS-2 during outburst.

\begin{tabular}{ccc|ccc}
\hline \hline $\begin{array}{c}\text { JD } \\
(+2450000)\end{array}$ & $\begin{array}{c}R \\
(\mathrm{mag})\end{array}$ & $\begin{array}{c}I \\
(\mathrm{mag})\end{array}$ & $\begin{array}{c}\text { JD } \\
(+2450000)\end{array}$ & $\begin{array}{c}R \\
(\mathrm{mag})\end{array}$ & $\begin{array}{c}I \\
(\mathrm{mag})\end{array}$ \\
\hline 2198 & $17.98 \pm 0.01$ & $17.86 \pm 0.04$ & 2221 & - & $18.99 \pm 0.08$ \\
2202 & $17.74 \pm 0.02$ & $17.72 \pm 0.02$ & 2222 & $18.53 \pm 0.03$ & $18.75 \pm 0.05$ \\
2203 & $18.04 \pm 0.02$ & $17.98 \pm 0.04$ & 2225 & $18.93 \pm 0.03$ & $19.11 \pm 0.06$ \\
2206 & $18.26 \pm 0.03$ & $18.23 \pm 0.03$ & 2226 & $18.67 \pm 0.03$ & - \\
2207 & $18.40 \pm 0.02$ & - & 2229 & - & $19.28 \pm 0.06$ \\
2208 & $18.11 \pm 0.02$ & - & 2230 & $19.03 \pm 0.03$ & $19.41 \pm 0.07$ \\
2213 & $18.53 \pm 0.03$ & - & 2231 & $18.83 \pm 0.04$ & $19.00 \pm 0.07$ \\
2216 & $18.43 \pm 0.03$ & $18.48 \pm 0.05$ & 2232 & $19.05 \pm 0.03$ & $19.30 \pm 0.08$ \\
2217 & $18.42 \pm 0.02$ & $18.61 \pm 0.07$ & 2236 & $19.18 \pm 0.03$ & - \\
2218 & $18.16 \pm 0.03$ & $18.20 \pm 0.04$ & 2237 & $19.16 \pm 0.04$ & $19.48 \pm 0.15$ \\
\hline
\end{tabular}

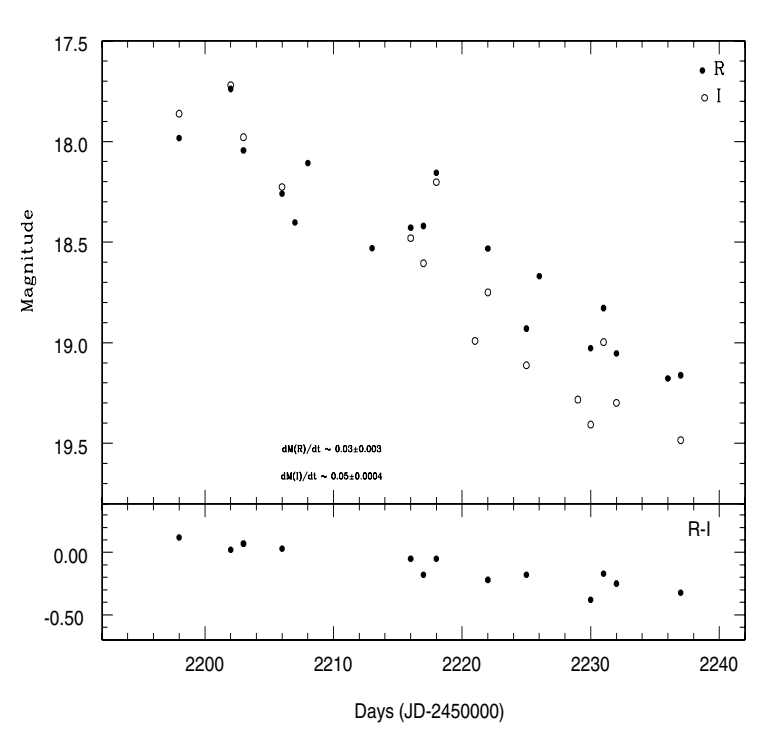

Fig. 4. $R, I$ light curve of the nova NMS-2 during outburst is shown in upper panel where filled and open circles represent $R$ and $I$ magnitudes respectively. The colour variation of the nova NMS-2 is shown in the lower panel.
The decay rate for the nova NMS-2 during our observation period is estimated to be:

$\frac{\mathrm{d} M(R)}{\mathrm{d} t} \sim 0.03 \pm 0.003 \mathrm{mag} \mathrm{day}^{-1}$

$\frac{\mathrm{d} M(I)}{\mathrm{d} t} \sim 0.05 \pm 0.004 \mathrm{mag}_{\mathrm{day}}{ }^{-1}$

for $R$ and $I$ bands respectively. The small value of the decay rate for the nova NMS-2 suggests that either it was a slow nova or, most likely, we observed it very late after it reached its peak brightness.

A re-brightening in the light curve of the nova NMS-2 from JD $\sim 2452210$ to 2452220 is evident in both $R$ and $I$ bands. This type of re-brightening profile in nova evolution is quite rare but not unique (Bonifacio et al. 2000). However, due to insufficient data points, we are not able to draw any firm conclusion about its exact behaviour. The evolution of $(R-I)$ colour for the nova NMS-2 is shown in the lower panel of Fig. 4. It becomes bluer with time which is consistent with normal nova behaviour. However, scattering in the data due to poor flat fielding at the edge of frames prevents us from drawing any conclusion about the dust formation in the ejecta. 


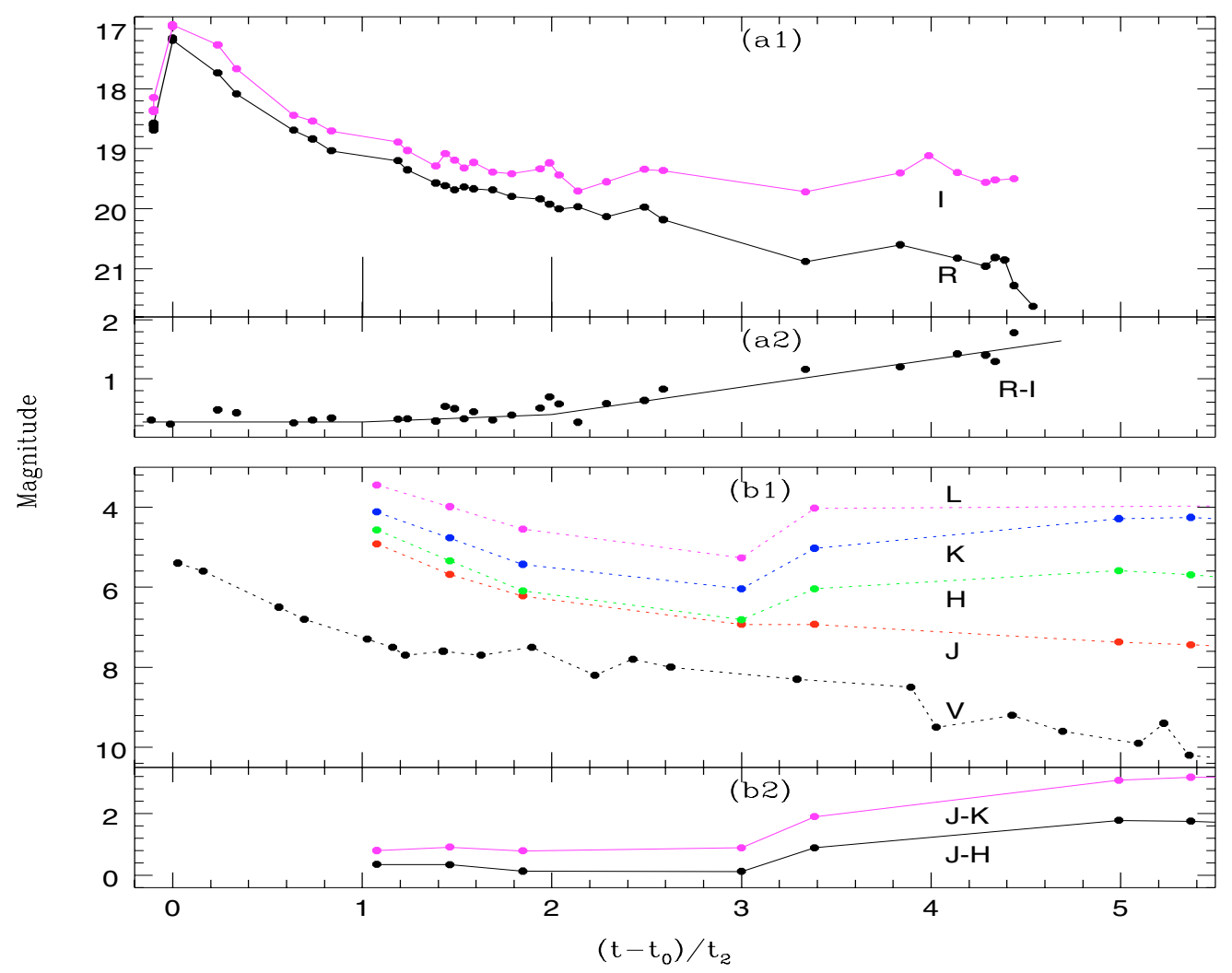

Fig. 5. $R, I$ light curves of the nova NMS-1 and its colour variation during the outburst is shown in the upper panels a1) and a2) respectively. VJHKL data along with the colour variation of the nova Herculis 1991, taken from the literature, is plotted in the lower panels b1) and b2) respectively, to compare the behaviour of the two novae. Here $t, t_{0}$ and $t_{2}$ indicate the time of observation, time of the maximum brightness and rate of decline respectively.

The detection rate of novae in M 31 is $~ 30$ novae per year (Arp 1956; Capaccioli et al. 1989; Shafter \& Irby 2001) and 2 novae detected in our survey, keeping our field of view and total observing time in mind, is consistent with these estimates.

\section{Discussion}

The $R$ and $I$ magnitudes of the nova NMS-1 at the brightest phase are estimated to be -7.96 and -8.02 mag respectively with a rate of decline of $\sim 0.11 \mathrm{mag}_{\mathrm{day}}{ }^{-1}$. A correlation of the rate of decline with the observed peak flux is in good agreement with the maximum magnitude versus rate of decline (MMRD) curve given by Capaccioli et al. (1989) for M 31 novae.

It is instructive to analyse the light curves and colour index, to investigate the evolution of the remnant. From the observed light curve of the nova NMS-1 (Fig. 2), it is evident that the flux in $R$ and $I$ bands decline in a similar way for the first three weeks but the late time evolution is qualitatively different. A difference in the profile of the light curves in two bands is a valuable diagnostic of the physical processes operating in the ejecta. We have neither spectroscopic information nor infrared photometry, nevertheless, we might estimate the possible evolution of the expanding shell from the $R, I$ light curves.

To study the photometric behavior of the nova NMS-1, we divided the declining part of the light curve into three sections as shown in Fig. 5(a1). The initial rapid decline in the flux follows a similar pattern in $R$ and $I$ bands and the colour remains practically unchanged; essentially this is the phase where emission from a region of progressively lower effective radius is received. However, after about three weeks, the $(R-I)$ colour shows a gradual increase indicating that the region is cooling. After about 40 days, the $R$ band flux is still progressively declining similar to that observed for the nova Herculis 1991 (Harrison \& Stringfellow 1994) in the $V$ band. The $I$ band, however, probably shows a slight gradual increase in flux during this period; a rapid rise in the colour index of the nova is seen in Fig. 5(a2). A similar behaviour has been observed in the case of nova Herculis 1991 where an almost constant plateau is seen in $J, H, K$ and $L$ bands (see Figs. 5(b1) and 5(b2)). Although, we have neither sufficient observations in the later stage nor supporting spectroscopy, we still believe that probably there is a signature of the formation of neutral hydrogen. The decrease in $R$ band flux but the reverse trend in $I$ band flux is likely to be due to the $\mathrm{H}_{\alpha}$ absorption and reradiation in longer wavelengths. There is some reason to speculate this because: a) we see a nearly constant peak Balmer flux of $10^{37} \mathrm{ergs} / \mathrm{s}$ declining rapidly, b) moderately large velocity gradient in the shell is shown by Balmer lines and c) A Balmer jump in the spectra (cf. Downes et al. 2001) are seen. If such a scenario can be confirmed, it could provide a diagnostic of dust formation during the expansion of wind in the nova.

For the nova NMS-2, we do not have light curve measurements in the initial phase and hence the epoch of peak 
brightness cannot be estimated. Unlike the nova NMS-1 where we had observations for about three months, the nova NMS-2 could be monitored only for about 40 days and during this period, $R$ and $I$ flux show a similar trend, except that $I$ declines faster. This is consistent with emission coming from a region of lower radius but higher temperature. Probably, the flux in both bands shows a bump similar to that observed in some novae.

What does our light curves imply about the formation of dust in the nova wind? Novae are believed to be a source of dust enrichment in the interstellar medium. However, there are two conflicting views (cf. Bode \& Evans, chap. 9): (1) Graphite or silicate grains can be formed in the cooling wind of nova depending on the chemical composition of white dwarf. (2) The nova environment could be hot and might not be the conducive of dust formation, in which case dust grains already present in the shell could cause an infra-red excess. If the expanding cool wind in the nova can produce grains, we should be able to see the reduction in temperature followed by an infra-red excess. A good monitoring program that tracks the temperature, luminosity as well as possible spectroscopy will be helpful in settling the question. Our limited $R, I$ observations suggest that the possible neutral hydrogen formation, when $(R-I)$ increases to about 1 mag from near zero, could act to shield dust grains against evaporation. This might be the reason for the slow increase in $I$ band flux despite the drop in $R$ band flux which is contributed in part by regions bluewards of the Balmer alpha line.

\section{Conclusion}

We found two novae in our survey carried out during 1998 to 2001 for an $\sim 13^{\prime} \times 13^{\prime}$ field belonging to the M 31 disk population. The rate of decline of nova NMS-1 suggests that it was a fast nova while the decay rate for nova NMS-2 suggests that either it was a slow nova or we observed it at a very late stage after the maximum brightness phase. The nova NMS-1 becomes cooler with time about six weeks after the maximum brightness phase accompanied by a slow increase in $I$ band flux, although the $R$ band flux still continues to decrease. This is in contrast with the normal behaviour of the novae. We suspect a secondary bump in $R, I$ light curves of the nova NMS-2, which is not unusual for normal nova evolution.
Acknowledgements. We are grateful to Stéphane Paulin-Henriksson for his help. We thank the anonymous referee for the useful comments. This study is a part of the project 2404-3 supported by Indo-French center for the Promotion of Advanced Research, New Delhi.

\section{References}

Arp, H. 1956, AJ, 61, 15

Baillon, P., Bouquet, A., Kaplan, J., \& Giraud-Héraud, Y. 1993, A\&A, 277,1

Bode, M. F., \& Evans, A. 1989, Classical Novae, Chap. 9 (Publisher, John Willy \& Sons Ltd)

Bonifacio, P., Selvelli, P. L., \& Caffau, E. 2000, A\&A, 356, L53

Ciardullo, R., Ford, H. C., Neil, J. D., Jacoby, G. H., \& Shafter, A. W. 1987, ApJ, 318, 520

Capaccioli, M., Della Valle, M., D’Onofrio, M., \& Rosino, L. 1989, AJ, 97, 1622

Donato, L., Garzvia, S., Conano, V., Sostero, G., \& Korlevic, K. 2001, IAUC, 7516

Downes, R. A., Duerbeck, H. W., \& Delahodde, C. E. 2001, JAD, 7, 6

Harrison, T. E., \& Stringfellow, G. S. 1994, ApJ, 437, 827

Hubble, E. 1929, ApJ, 69, 103

Jacoby, C. H., Branch, D. G., Ciardullo, R., et al. 1992, PASP, 104, 599

Joshi, Y. C., Pandey, A. K., Narasimha, D., \& Sagar, R. 2001, BASI, 29,531

Joshi, Y. C., Pandey, A. K., Narasimha, D., Sagar, R., \& Giraud-Héraud, Y. 2003a, A\&A, 402, 113

Joshi, Y. C., Pandey, A. K., Narasimha, D., \& Sagar, R. 2003b, BASI, presented in XXII ASI Meeting, Feb. 13-15, 2003

Joshi, Y. C., et al. 2003c, under preparation

Landolt, A. U. 1992, AJ, 104, 340

Li, W. D. 2001, IAUC, 7729

McLaughlin, D. B. 1945, PASP, 57, 69

Rector, T. A., Jacoby, G. H., Corbett, D. L., Denham, M., RBSE Nova Search Team 1999, BAAS, 195, 3608

Rosino, L. 1964, A\&A, 27, 498

Rosino, L. 1973, A\&AS, 3, 347

Rosino, L., Capacciol, M., D’Onofrio, M., \& Della Valle, M. 1989, AJ, 97, 83

Sharov, A. S., \& Alksnis, A. 1991, ApSS, 180, 273

Shafter, A. W., \& Irby, B. K. 2001, ApJ, 563, 749

Stetson, P. B. 1987, PASP, 99, 191

Tomaney, A. B., \& Shafter, A. W. 1992, ApJS, 81, 683

van den Bergh, S. 1988, PASP, 100, 1486 